\title{
Utility of Draining Fluid Collections Following Mesh Ventral Hernia Repair
}

\author{
Maureen P. Kohi ${ }^{\star}$, Ryan Kohlbrenner, Evan Lehrman, Andrew G. Taylor, Kanti P. Kolli, Robert K. Kerlan Jr and Nicholas \\ Fidelman \\ Department Department of Radiology and Biomedical Imaging, University of California, 505 Parnassus Avenue, M-361, San Francisco, CA
} 94143, USA

\section{Abstract}

Background: Ventral hernia repair (VHR) is one of the most common surgical procedures and is commonly associated with post procedural fluid collection formation.

Purpose: To determine the clinical outcomes of percutaneous drainage of collections adjacent to mesh ventral hernia repair (VHR).

Material and Methods: A retrospective review of all consecutive patients who underwent percutaneous drainage of fluid collections adjacent to mesh VHR was performed. Patient characteristics, mesh type, culture results, and clinical outcomes were reviewed. Clinical success was defined as mesh salvage and resolution of presenting signs and symptoms without surgical intervention.

Results: A total of 14 patients were included (6 men, 8 women) with median age of 53 years (range: 2280 years). Median interval time between VHR and drain placement was 40 days (range: 15-1144 days). Pain was the most common presenting symptom, noted in 13 patients $93 \%$ ), followed by erythema which was noted in 10 patients (71\%). Percutaneous drain insertion was technically successful in all 14 patients (100\%). Clinical success was achieved for five out of 14 patients (36\%) whose mesh was salvaged. The other nine patients (64\%) required mesh excision because of lack of clinical improvement. There was no statistically significant difference in drainage outcomes when comparing patient age, gender, mesh type, interval from surgery to drainage, or bacterial cultures.

Conclusion: While percutaneous drainage of collections adjacent to mesh VHR may be successful in some cases, a majority of patients may ultimately require surgical mesh excision.

\section{Introduction}

Ventral hernia repair (VHR) is one of the most common procedures performed by general surgeons [1]. Each year, more than 350,000 VHRs are performed in the United States [2]. While usually considered a relatively low-risk procedure, VHR is associated with a morbidity rate of up to $60 \%$ with complications including wound infection, seroma formation, reoperation, hospital readmission, and cardiovascular abnormalities [3].

In order to reduce the risk of hernia recurrence, which is associated with the conventional suture repair technique, mesh repair has become a popular alternative for VHR [4]. While mesh repair results in fewer cases of hernia recurrences, the rate of seroma formation and surgical site infection is higher compared with suture repair [4].

Despite sterile technique and pre-procedural antibiotics, $8 \%$ of all VHRs are complicated by infection [5]. Several therapeutic options are available for treatment of post mesh repair infection, including complete or partial surgical excision, percutaneous drainage, and negative pressure therapy [5]. Percutaneous drainage of collections associate with mesh repair is an attractive option as it is a minimally invasive procedure with the theoretical benefit of treating the infection without surgical excision of the mesh. The evidence behind non-operative management of mesh-associated fluid collections has been limited to date [6-8]. Therefore, the purpose of this study was to evaluate the effectiveness of percutaneous drainage of collections adjacent to mesh VHR in symptomatic patients at our institution.

\section{Materials and Methods}

\section{Patients}

This study was approved by the Committee on Human Research of

\section{Publication History:}

Received: January 04, 2017

Accepted: March 25, 2017

Published: March 27, 2017

Keywords:

Fluid collections, Ventral hernia repair the Institutional Review Board at our institution. Informed consent requirement was waived. A retrospective record review of all consecutive patients who underwent percutaneous drainage of fluid collections adjacent to mesh VHR at our institution between June 2000 and December 2013 was performed. A total of 14 patients were included ( 6 men, 8 women) with median age of 53 years (range: $22-80$ years). Patients undergoing fluid aspiration without drain placement or undergoing drainage of fluid collections not adjacent to the mesh in the abdominal fascial layer were excluded. Additionally, cases of existing surgical drainage catheters presenting for tube changes were excluded. Patient demographics, operative reports, clinical notes, and laboratory data were obtained through the electronic medical records. Images before and after the drainage procedures were reviewed using the picture archiving and communication system.

\section{Percutaneous drainage procedure}

All procedures were performed by fellowship trained interventional radiologists. The drainage procedures were performed under sonographic or computed tomographic (CT) guidance with moderate sedation. Following sterile preparation and local anesthesia with $1 \%$

"Corresponding Author: Dr. Maureen P. Kohi, Department Department of Radiology and Biomedical Imaging, University of California, 505 Parnassus Avenue, M-361, San Francisco, CA 94143, USA; Tel: +(415) 353-1300; E-mail: maureen.kohi@ucsf.edu

Citation: Kohi MP, Kohlbrenner R, Lehrman E, Taylor AG, Kolli KP, et al. (2017) Utility of Draining Fluid Collections Following Mesh Ventral Hernia Repair. Int J Surg Surgical Porced 2: 116. doi: https://doi.org/10.15344/2456-4443/2017/116

Copyright: @ 2017 Kohi et al. This is an open-access article distributed under the terms of the Creative Commons Attribution License, which permits unrestricted use, distribution, and reproduction in any medium, provided the original author and source are credited. 
Citation: Kohi MP, Kohlbrenner R, Lehrman E, Taylor AG, Kolli KP, et al. (2017) Utility of Draining Fluid Collections Following Mesh Ventral Hernia Repair. Int J Surg Surgical Porced 2: 116. doi: https://doi.org/10.15344/2456-4443/2017/116

Page 2 of 7

lidocaine, a 21-gauge or a 19-gauge needle was used to access the collection. In case of a 21-gauge needle entry choice, a 0.018 - inch guidewire and a 4-French (F) micropuncture conversion system (Cook, Inc, Bloomington, Indiana, USA) were used. Subsequently, a 0.035 -inch guidewire was inserted through the 4 -F introducer. If a19gauge needle was used, a 0.035 -inch guidewire was inserted directly through the needle. Guidewire placement within the collection was confirmed with sonographic or CT imaging. Following serial dilatations, the drainage catheter was placed into the collection. The size and type of the drainage catheter was at the discretion of the treatinginterventional radiologist. Multipurpose or Dawson-Mueller (Cook, Inc, Bloomington, Indiana, USA) drainage catheters ranging from $8.5-\mathrm{F}$ to $12.0-\mathrm{F}$ were used. Following drain placement, as much fluid as possible was aspirated from the catheter and a sample was sent for bacterial culture and Gram Stain evaluation.

\section{Study outcomes}

Study outcomes were technical success and clinical success of percutaneous drainage of fluid collections adjacent to the mesh VHR. Technical success was defined as successful insertion of a percutaneous drainage catheter into the target collection. Clinical success was defined as mesh salvage and resolution of presenting signs and symptoms without surgical intervention. Mesh excision was defined as surgical removal of the mesh despite percutaneous drainage.

\section{Statistical analysis}

The variables examined in the study included patient age, gender distribution, interval from surgery to drain placement, mesh type, drain diameter and type, volume of fluid removed during drain placement, and results of microbiological fluid analysis. The data were analyzed using the Fisher's exact test and the Wilcoxon rank sum test. A $p$ value $<0.05$ was considered statistically significant.

\section{Results}

Pain was the most common presenting symptom, noted in 13 patients $(86 \%)$, and followed by erythema, which was present in 10 patients $(71 \%)$. Other symptoms included fever present in four patients (29\%) and nausea noted in two patients (14\%). Median interval time between VHRand drain placement was 40 days (range: 15-1144 days). Patient demographics are presented in Table 1. A variety of mesh types was used for the VHR procedures which were performed laparoscopically; 6 patients $(43 \%)$ had polypropylene (PP), 3 patients (21\%) had polytetrafluoroethylene (PTFE), 2 patients (14\%) had porcine dermal collagen (PDC), and the remaining 3 had either a combination of PP and PTFE or polyester mesh, (Table 2).

Sonographic guidance was used in 8 patients (57\%) and CT guidance was used in the remaining 6 patients (43\%) to gain entry into the fluid collection. A 19-gauge needle was used in 12 patients (86\%) and a 21 -gauge needle was used in the remaining 2 patients (14\%). Drain placement varied between patients; 7 patients $(50 \%)$ received a $12-\mathrm{F}$ multipurpose drain, 2 patients (14\%) received a 10-F multipurpose drain, and 5 patients (36\%) received an 8.5-F Dawson-Muller drain. The median amount of fluid removed at the time of the procedure was $35 \mathrm{ml}$ (range: $10-750 \mathrm{ml}$ ). Microbiology evaluation of the fluid sent for analysis was positive for bacterial culture in 10 patients (71\%). Six patients (43\%) were diagnosed with Staphylococcus aureus, 2 patients (14\%) had infection with Pseudomonas aeruginosa, and the

\begin{tabular}{|l|l|l|l|l|}
\hline $\begin{array}{l}\text { Patient } \\
\text { Number }\end{array}$ & $\begin{array}{l}\text { Age } \\
\text { (years) }\end{array}$ & Gender & $\begin{array}{l}\text { Presenting } \\
\text { Symptoms }\end{array}$ & $\begin{array}{l}\text { Interval from } \\
\text { Surgery } \\
\text { to Drainage (days) }\end{array}$ \\
\hline 1 & 48 & $\mathrm{~F}$ & $\mathrm{P}$ & 412 \\
\hline 2 & 69 & $\mathrm{M}$ & $\mathrm{P} / \mathrm{E}$ & 37 \\
\hline 3 & 54 & $\mathrm{~F}$ & $\mathrm{P} / \mathrm{N} / \mathrm{V} / \mathrm{E}$ & 24 \\
\hline 4 & 52 & $\mathrm{~F}$ & $\mathrm{P} / \mathrm{N} / \mathrm{F} / \mathrm{E}$ & 18 \\
\hline 5 & 79 & $\mathrm{~F}$ & $\mathrm{P}$ & 27 \\
\hline 6 & 45 & $\mathrm{~F}$ & $\mathrm{P} / \mathrm{E}$ & 671 \\
\hline 7 & 22 & $\mathrm{~F}$ & $\mathrm{P} / \mathrm{E}$ & 15 \\
\hline 8 & 57 & $\mathrm{M}$ & $\mathrm{P} / \mathrm{F} / \mathrm{E}$ & 1144 \\
\hline 9 & 57 & $\mathrm{~F}$ & $\mathrm{P} / \mathrm{F}$ & 51 \\
\hline 10 & 51 & $\mathrm{M}$ & $\mathrm{E}$ & 35 \\
\hline 11 & 80 & $\mathrm{M}$ & $\mathrm{P} / \mathrm{E}$ & 342 \\
\hline 12 & 42 & $\mathrm{M}$ & $\mathrm{P} / \mathrm{F} / \mathrm{E}$ & 43 \\
\hline 13 & 44 & $\mathrm{M}$ & $\mathrm{P} / \mathrm{E}$ & 31 \\
\hline 14 & 71 & $\mathrm{~F}$ & $\mathrm{P}$ & 64 \\
\hline
\end{tabular}

Table 1: Patient Demographics.

F: Female, M: Male, P: Pain, E: Erythema, N: Nausea, V: Vomiting, F: Fever

remaining 2 patients (14\%) had positive cultures for Bacteroidesfragilis and Enterobacter cloacae. Microbiology analysis did not yield organisms in the remaining four patients.

Percutaneous drain insertion was technically successful in all 14 patients (100\%). Clinical success was noted in five out of 14 patients (36\%) in whom the mesh was salvaged. In all five patients, the drains were in place for a median of 7 days (range: 5-21 days) and removed by either interventional radiology ( 3 patients) or by surgery in outpatient follow-up clinic ( 2 patients). Mesh salvage was not possible in 9 out of the 14 patients (64\%), who ultimately had mesh excision. The interval from percutaneous drainage to mesh excision varied from 7 days to 1-year following drainage. Two patients underwent excision 7 -days following drainage due to persistent infection and pain. There was no statistically significant difference in drainage outcome when comparing patient age, gender, mesh type, interval from surgery to drainage, drain type, or positive bacterial cultures.

\section{Discussion}

The use of mesh for VHR has increased from approximately $30 \%$ in 1987 to over 65\% in 1999 [9] as clinical trials have demonstrated mesh repair to be associated with a lower recurrence rate [4]. While mesh VHR has become the standard of care [10], it is associated with more seroma formations and surgical site infections [11] compared to suture repair. Mesh infection is a dreaded complication of prosthetic hernia repair with a challenging management [12]. In most cases, surgical excision of the mesh is performed which may result in delayed repair of the hernia with an alternative surgical strategy and potential for hernia recurrence [12].

Several reports have discussed mesh salvage through conservative measures such as intravenous or topical antibiotics, incision and drainage, and negative pressure therapy [6-8]. Greenberg [6] reported successful mesh salvage in seven out of 11 patients with meshrelated infections using conservative measures such as local wound 
Citation: Kohi MP, Kohlbrenner R, Lehrman E, Taylor AG, Kolli KP, et al. (2017) Utility of Draining Fluid Collections Following Mesh Ventral Hernia Repair. Int J Surg Surgical Porced 2: 116. doi: https://doi.org/10.15344/2456-4443/2017/116

Page 3 of 7

\begin{tabular}{|c|c|c|c|c|c|}
\hline Patient Number & Mesh Type & Drain Type & Volume of Fluid Removed (ml) & Culture Results & Clinical Outcome \\
\hline 1 & $\mathrm{PP}$ & $10-\mathrm{F}, \mathrm{M}$ & 500 & P. aeruginosa & Excision 1-year after drainage \\
\hline 2 & PTFE & 12-F, M & 500 & No growth & Excision 7-days after drainage \\
\hline 3 & PTFE & 8.5-F, DM & 15 & S. aureus & Excision 16-days after drainage \\
\hline 4 & $\mathrm{PP}$ & 8.5-F, DM & 20 & P. aeruginosa & Excision 7-days after drainage \\
\hline 5 & PDC & $12-\mathrm{F}, \mathrm{M}$ & 10 & E. cloacae & Excision 5 months after drainage \\
\hline 6 & $\mathrm{PTFE} / \mathrm{PP}$ & 12-F, M & 25 & S. aureus & Excision 21-days after drainage \\
\hline 7 & Polyester & $12-\mathrm{F}, \mathrm{M}$ & 140 & S. aureus & Excision 11 months after drainage \\
\hline 8 & $\mathrm{PTFE} / \mathrm{PP}$ & 8.5-F, DM & 750 & No growth & Excision 7 months after drainage \\
\hline 9 & $\mathrm{PP}$ & 8.5-F, DM & 30 & No growth & Excision 10 months after drainage \\
\hline 10 & $\mathrm{PP}$ & 8.5-F, DM & 30 & S. aureus & Excision 12 months after drainage \\
\hline 11 & $\mathrm{PP}$ & 12-F, M & 70 & S. aureus & Salvage \\
\hline 12 & PTFE & 12-F, M & 40 & No growth & Salvage \\
\hline 13 & $\mathrm{PP}$ & 10-F, M & 10 & S. aureus & Salvage \\
\hline 14 & PDC & 12-F, M & 10 & B. fragilis & Salvage \\
\hline
\end{tabular}

Table 2: Drainage Outcomes.

PP: Polypropylene, PTFE: Polytetrafluoroethylene, PDC: Porcine dermal collagen, F: French, M: Multipurpose, DM: Dawson-Mueller

debridement, partial mesh excision, wound vacuum, and antibiotics. Four of 11 patients ultimately had mesh excision. Greenberg concluded that in the absence of extensive mesh infection or sepsis, infected mesh may be salvaged. However, treatment of complex patients should be individualized. Trunzo et al. [7] report mesh salvage in two patients using parenteral antibiotics, percutaneous drainage, and gentamicin irrigations via the drain. The authors concluded that percutaneous drainage followed by antibiotic irrigation is a potential alternative to mesh excision.

Our study evaluated the role of percutaneous drainage of fluid collections adjacent to mesh VHR. We observed that drain placement was only clinically successful in five out of 14 patients (36\%). In fact, the majority of patients (9 out of 14,64\%) had mesh excision. Our findings are in contrast to the findings of Kuo et al. [13], who showed that percutaneous drainage of mesh-related collections was successful in 16 out of $21(76 \%)$ of patients treated with percutaneous drainage. One potential explanation for Kuo et al's higher rate of mesh salvage is the numerous tube checks and changes performed. In that study, following percutaneous drain placement, several catheter checks were performed ranging from one to 11 times, with a mean of four. This is compared to our study where catheter checks and exchanges were performed only on five patients with a median of 2, ranging from one to 3. Continual and vigilant catheter surveillance with repeated tube checks and changes may potentially improve fluid drainage and result in better mesh salvage rate and reduce re-operation rates.

Another possible explanation for the difference in the mesh salvage rate is the superficial location of the fluid collections in Kuo et al's series [13]. In their study, 14 out of 21 collections were in the subcutaneous layer, making them more accessible to percutaneous drainage with less mesh involvement. We only included patients with fluid collections that involved the mesh and therefore were fasciainvolving.

In contrast to the findings of Trunzo et al. [7], we did not perform antibiotic irrigation through the percutaneous drain. Perhaps antibiotic irrigation with gentamicin over a course of several weeks as demonstrated by Trunzo et al could result in higher rates of mesh salvage.

Similar to the findings of Kuo et al. [13], fluid culture revealed a wide spectrum of pathogens with S. aureus being the most common pathogen isolated from the fluid. Culture did not predict mesh excision. We did not find that the type of mesh predicted surgical excision.

Our study is limited by its retrospective design. The overall incidence of mesh-related infections at our institution is unknown and we only analyzed symptomaticpatients who were referred to us for percutaneous drainage. Additionally, this was a single institutional review. Different institutions might have various approaches to management of fluid collections adjacent to the mesh VHR. Patietn comorbidities could also affect the outcomes of mesh VHR. The lack of a control group with patients undergoing surgery or watchful waiting may have affected our conclusions. Lastly, the small sample size prevented us from performing meaningful subgroup analyses.

In conclusion, while conservative management of mesh-related fluid collections following VHR may result in mesh salvage, almost $2 / 3$ of our patients ultimately had mesh excision. Our findings highlight the varying efficacy of conservative management of meshrelated collections. Further research is required to determine the effectiveness of percutaneous drainage for mesh salvage as compared to surgical excision. Until that time, percutaneous drainage of meshrelated fluid collections should be performed on a case-by-case basis with the foresight that continual tube surveillance may improve the success of the drainage technique. Additionally, if the collections are superficial, percutaneous drainage may result in mesh salvage and prevent surgery. However, if repeated catheter checks and changes cannot be performed, percutaneous drainage may not salvage the mesh and surgical excision may be a more appropriate management for that clinical setting.

\section{Competing Interests}

The authors declare that they have no competing interests. 
Citation: Kohi MP, Kohlbrenner R, Lehrman E, Taylor AG, Kolli KP, et al. (2017) Utility of Draining Fluid Collections Following Mesh Ventral Hernia Repair. Int J Surg Surgical Porced 2: 116. doi: https://doi.org/10.15344/2456-4443/2017/116

\section{References}

1. Brahmbhatt R, Carter SA, Hicks SC, Berger DH, Liang MK (2014) Identifying risk factors for surgical site complications after laparoscopic ventral hernia repair: evaluation of the ventral hernia working group grading system. Surg Infect 15: 187-193.

2. Poulose BK, Shelton J, Phillips S, Moore D, Nealon W (2012) Epidemiology and cost of ventral hernia repair: Making the case for hernia research. Hernia 16:179-183

3. Bellows CF, Robinson C, Fitzgibbons RJ, Webber LS, Berger DH, et al (2014) Watchful waiting for ventral hernias: a longitudinal study. Am Surg 80: $245-252$

4. Nguyen MT, Berger RL, Hicks SC, Davila JA, Li LT, et al. (2014) Comparison of Outcomes of Synthetic Mesh vs Suture Repair of Elective Primary Ventral Herniorrhaphy: A Systematic Review and Meta-analysis. JAMA Surg 149: 415-421.

5. Berrevoet F, Vanlander A, Sainz-Barriga M, Rogiers X, Troisi R (2013) Infected large pore meshes may be salvaged by topical negative pressure therapy. Hernia 17: 67-73.

6. Greenberg JJ (2010) Can infected composite mesh be salvaged? Hernia14: 589-592.

7. Trunzo JA, Ponsky JL, Jin J, Williams CP, Rosen MJ (2009) A nove approach for salvaging infected prosthetic mesh after ventral hernia repair Hernia13: 545-549.

8. Berrevoet F, Vanlander A, Sainz-Barriga M, Rogiers X, Troisi R (2012) Infected large pore meshes may be salvaged by topical negative pressure therapy. Hernia 17: 67-73.

9. Chung L, Tse GH, O'Dwyer PJ (2014) Outcome of patients with chronic mesh infection following abdominal wall hernia repair. Hernia 18: 701-704.

10. Liang MK, Berger RL, Nguyen MT, Hicks SC, Li LT, et al. (2014) Outcomes with porcine acellular dermal matrix versus synthetic mesh and suture in complicated open ventral hernia repair. Surg Infect 15: 506-512.

11. Yabanoglu H, Arer I, Caliskan K (2015) The effect of the use of synthetic mesh soaked in antibiotic solution on the rate of graft infection in ventral hernias: a prospective randomized study. Int Surg 100: 1040-1047.

12. Hanna M, Dissanaike S (2015) Mesh ingrowth with concomitant bacteria infection resulting in inability to explant: a failure of mesh salvage. Hernia 19: 339-344.

13. Kuo YC, Mondschein JI, Soulen MC, Patel AA, Nemeth A, et al. (2010) Drainage of collections associated with hernia mesh: is it worthwhile? VascInterv Radiol 21: 362-366. 